\title{
Chemoradiotherapy-induced Changes in Mucinous Components in Rectal Cancer Tissue: Evaluation on High Iron Diamine-alcian Blue and Mucin 1 Staining
}

\author{
HIROSHI MIYAKITA ${ }^{1}$, SOTARO SADAHIRO ${ }^{1}$, YUTARO KAMEI $^{1}$, TAKASHI OGIMI ${ }^{1}$, GOTA SAITO ${ }^{1}$, \\ KAZUTAKE OKADA ${ }^{1}$, AKIRA TANAKA ${ }^{1}$, TOSHIYUKI SUZUKI ${ }^{1}$, HIROSHI KAJIWARA $^{2}$ and TAKESHI AKIBA ${ }^{3}$ \\ ${ }^{1}$ Department of Surgery, Tokai University, Kanagawa, Japan; \\ ${ }^{2}$ Department of Pathology, Tokai University, Kanagawa, Japan; \\ ${ }^{3}$ Department of Radiology, Tokai University, Kanagawa, Japan
}

\begin{abstract}
Background/Aim: The standard treatment for rectal cancer is neoadjuvant chemoradiotherapy ( $n C R T)$ followed by surgery. Mucinous carcinoma responds poorly to $n C R T$. In some patients, mucin lakes (MLs) are induced by $n C R T$. Identifying whether MLs are induced or originally present would be of great importance. Patients and Methods: We studied 20 patients with MLs (CRT-MC group) among 205 patients who received $n C R T$. Among 88 patients who did not receive $n C R T$, we studied 9 patients with mucinous carcinoma (non-CRT-MC group) and 18 patients with MLs in differentiated adenocarcinoma (nonCRT-AC group). Tumors were stained with high iron diamineAlcian blue (HID-AB) and MUC1 staining. Results: Rate of $A B>H I D$ staining of cancer cells was significantly higher in the $C R T-M C$ group than in non-CRT-MC group ( $p=0.0004)$. Rate of MUC1 staining in MLs was significantly higher in the CRT$M C$ group (p=0.0254). Conclusion: nCRT can induce qualitative changes in mucinous components, however, other methods are required to distinguish induced components from originally existing components.
\end{abstract}

Total mesorectal excision (TME) after neoadjuvant chemoradiotherapy (nCRT) is the standard treatment for rectal cancer (1-4). Patients with mucinous carcinoma have been reported to have a poor response to nCRT, as well as poor outcomes. In some patients, however, nCRT may induce the formation of mucin lakes (MLs) in rectal cancer

This article is freely accessible online.

Correspondence to: Sotaro Sadahiro, MD, Ph.D., Department of Surgery, Tokai University, 143 Shimokasuya Isehara, Kanagawa, 259-1193, Japan. Tel: +81 463931121, Fax: +81 463965577, e-mail: Sadahiro@is.icc.u-tokai.ac.jp

Key Words: Rectal cancer, chemoradiotherapy, mucinous carcinoma, mucous lake, MUC1, HID-AB. tissue (5-12). The ability to determine whether MLs are induced or originally exist in resected specimens would be of great clinical significance.

Sialomucin and sulphomucin are included in intracytoplasmic mucin of cancer cells and MLs in patients with colorectal cancer and can be identified by high iron diamine-Alcian blue (HID-AB) staining (13). Mucin 1 (MUC1), a transmembrane mucin and a membrane-bound glycoprotein, exists in cancer cells and in MLs in colorectal cancer tissue and has been reported to be related to outcomes (14-17).

HID-AB staining and MUC1 immunohistochemical staining was, therefore, performed in resected specimens of rectal cancer obtained from patients who received nCRT and those who did not, to evaluate mucinous components in cancer cells and MLs in cancer tissue and examine whether nCRT induces changes in mucinous components.

\section{Patients and Methods}

Subjects. From 2005 through 2015, a total of 293 patients with rectal cancer underwent curative surgery. Among the 205 patients who received nCRT, the ML formation which occupied at least $20 \%$ of the area of the maximum cut surface of the resected specimens was found in 20 patients. These patients were designated as the CRT-MC group. Among the 88 patients who did not receive CRT, 9 patients were histologically diagnosed with mucinous carcinoma on the resected specimens in which ML formation was remarkable (non-CRT-MC group), and 18 patients were diagnosed with well- or moderately differentiated adenocarcinoma but had apparent ML formation in less than $50 \%$ of the area ratios of the tumors. These patients were designated as the non-CRT-AC group. A total of 47 patients were studied. This study was approved by the institutional review board of our University (17R-238), and all patients gave their written informed consent.

HID-AB staining, MUC1 staining, and evaluations. To perform HID$\mathrm{AB}$ staining, the sections were deparaffinized and then stained with HID solution, prepared using N, N-dimethyl-m-phenylenediamine dihydrochloride $(120 \mathrm{mg}$ ) and N,N-dimethyl-p-phenylenediamine 
monohydrochloride $(20 \mathrm{mg})$ (Sigma-Aldrich Japan Inc., Osaka, Japan), and $\mathrm{pH} 2.5$ Alcian blue solution (Muto Pure Chemicals, Tokyo, Japan). For MUC1 staining, the sections were deparaffinized in xylene, and antigen activation was performed at $95^{\circ} \mathrm{C}$ for $76 \mathrm{~min}$, using pH 6.0 ULTRA CC1 buffer (Ventana Medical Systems Inc., Tucson, AZ, USA). After antigen activation, the sections were allowed to react with anti-MUC1 H23 (Ventana Medical Systems Inc.) for $40 \mathrm{~min}$. After HID-AB staining, the tumor cells and MLs were separately evaluated. Tumor cells or MLs predominantly stained by $\mathrm{AB}$ were classified as $\mathrm{AB}>\mathrm{HID}$, and those predominantly stained by HID were classified as HID $>A B$. The results of MUC1 staining were classified as positive or negative. The results of staining were evaluated separately by 2 physicians (HM and $\mathrm{HK}$ ). If different evaluations were obtained, microscopic examinations were repeated by the 2 physicians (HM, HK) to determine the results.

Statistical analysis. Chi-square tests were used to evaluate differences between the 2 groups. $p$-Values of less than 0.05 were considered to indicate statistical significance. Statistical analysis was performed with software package JMP10 (SAS institute Inc., Cary, NC, USA).

\section{Results}

Patient characteristics. Table I shows the patient characteristics. The proportion of women was higher in the subgroup of patients with MLs in differentiated adenocarcinoma (non-CRT-AC group) than in the other 2 groups. In the CRT-MC group, the proportion of patients with lower rectal cancer was significantly higher than that in the 2 non-CRT groups.

HID-AB staining (Table II, Figure 1). As for the results of staining for tumor cells, the percentage of tumor cells predominantly stained with $\mathrm{AB}$ was significantly higher in the CRT-MC group than in the non-CRT group $(p=0.0004)$. The results of staining for MLs did not differ between the CRT-MC group and the non-CRT group $(p=0.6889)$. The results of HID-AB staining for tumor cells and MLs did not differ between the CRT-MC group and the non-CRT group $(p=0.1939$ and $p=0.1661)$.

MUC1 staining (Table III, Figure 2). The rate of positive staining for tumor cells did not differ between the CRT-MC group and the non-CRT group ( $p=0.4039)$. On comparing the non-CRT-AC group with the non-CRT-MC group, the rate of positive staining for tumor cells was significantly higher in the non-CRT-AC group ( $p=0.0377)$. The rate of positive staining for MLs was significantly higher in the CRT-MC group (70\%) than in the non-CRT-MC group $(37 \%, p=0.0254)$.

\section{Discussion}

Patients with mucinous carcinoma have been reported to poorly respond to nCRT, resulting in poor outcomes. In some of the patients, however, MLs are induced by nCRT in rectal
Table I. Patient characteristics.

\begin{tabular}{|c|c|c|c|c|}
\hline Variable & $\begin{array}{c}\text { CRT-MC } \\
\text { n }(\%)\end{array}$ & $\begin{array}{c}\text { MC } \\
\mathrm{n}(\%)\end{array}$ & $\begin{array}{c}\mathrm{AC} \\
\mathrm{n}(\%)\end{array}$ & $p$-Value \\
\hline \multicolumn{5}{|l|}{ Gender } \\
\hline Male & $17(85.0)$ & $7(77.8)$ & $8(44.4)$ & \\
\hline Female & $3(15.0)$ & $2(22.2)$ & $10(55.6)$ & 0.0218 \\
\hline \multicolumn{5}{|l|}{ Age (years) } \\
\hline Range & $35-82$ & $36-81$ & $40-86$ & \\
\hline Median & 61.9 & 63.4 & 65.8 & 0.6663 \\
\hline \multicolumn{5}{|l|}{ Location of tumor } \\
\hline Upper rectum & $7(35.0)$ & $6(66.7)$ & $11(61.1)$ & \\
\hline Lower rectum & $13(65) .0$ & $3(33.3)$ & 7 (38.9) & 0.0069 \\
\hline \multicolumn{5}{|l|}{ Surgical Procedure } \\
\hline LAR & $13(65.0)$ & $6(66.7)$ & $16(88.9)$ & \\
\hline Hartmann & $1(5.0)$ & 0 & $2(11.1)$ & \\
\hline APR & $5(25.0)$ & $2(22.2)$ & 0 & \\
\hline Total colectomy & 0 & $1(11.1)$ & 0 & \\
\hline Local excision & $1(5.0)$ & 0 & 0 & 0.1082 \\
\hline \multicolumn{5}{|l|}{$\mathrm{T}$ classification } \\
\hline 0 & 0 & 0 & 0 & \\
\hline 1 & $2(10.0)$ & 0 & $1(5.6)$ & \\
\hline 2 & $3(15.0)$ & 0 & 0 & \\
\hline 3 & $12(60.0)$ & $7(77.8)$ & 17 (94.4) & \\
\hline 4 & $3(15.0)$ & $2(22.2)$ & 0 & 0.2106 \\
\hline \multicolumn{5}{|l|}{$\mathrm{N}$ classification } \\
\hline+ & $7(35.0)$ & $3(33.3)$ & $10(55.6)$ & \\
\hline- & $13(65.0)$ & $6(66.7)$ & $8(44.4)$ & 0.3634 \\
\hline \multicolumn{5}{|l|}{ ly } \\
\hline+ & $10(50.0)$ & $6(66.7)$ & $12(66.7)$ & \\
\hline- & $10(50.0)$ & $3(33.3)$ & $6(33.3)$ & 0.5155 \\
\hline \multicolumn{5}{|l|}{$\mathrm{v}$} \\
\hline+ & $11(55.0)$ & $5(55.6)$ & $13(72.2)$ & \\
\hline- & $9(45.0)$ & $4(44.4)$ & $5(27.8)$ & 0.5048 \\
\hline \multicolumn{5}{|l|}{ (ур) Stage } \\
\hline 1 & $2(10.0)$ & 0 & 0 & \\
\hline 2 & $12(60.0)$ & $6(66.7)$ & $8(44.4)$ & \\
\hline 3 & $7(35.0)$ & $3(33.3)$ & $10(55.6)$ & 0.5271 \\
\hline
\end{tabular}

LAR: Low anterior resection; APR: abdominoperineal resection; Ly: lymphatic invasion; v: venous invasion.

cancer tissue (5-12). In patients who receive nCRT, the incidence of mucinous carcinoma is relatively high, ranging from $13 \%$ to $24 \%(6,8,11)$. Patients in whom MLs are induced have been reported to have better outcomes than patients who originally have MLs (12). However, the nCRTinduced qualitative changes in the components of MLs remain to be confirmed. We therefore examined whether nCRT induces qualitative changes in the mucinous components of cancer cells and cancer stromal tissue.

HID-AB staining is a dual staining technique for identifying intracytoplasmic mucin of cancer cells. Sialomucin is stained blue, and sulphomucin is stained black. A mixture of neutral and acidic mucins is present in most intracytoplasmic mucin of cancer cells. Acidic mucin is a mixture of sialomucin and sulphomucin. MLs are usually 
a

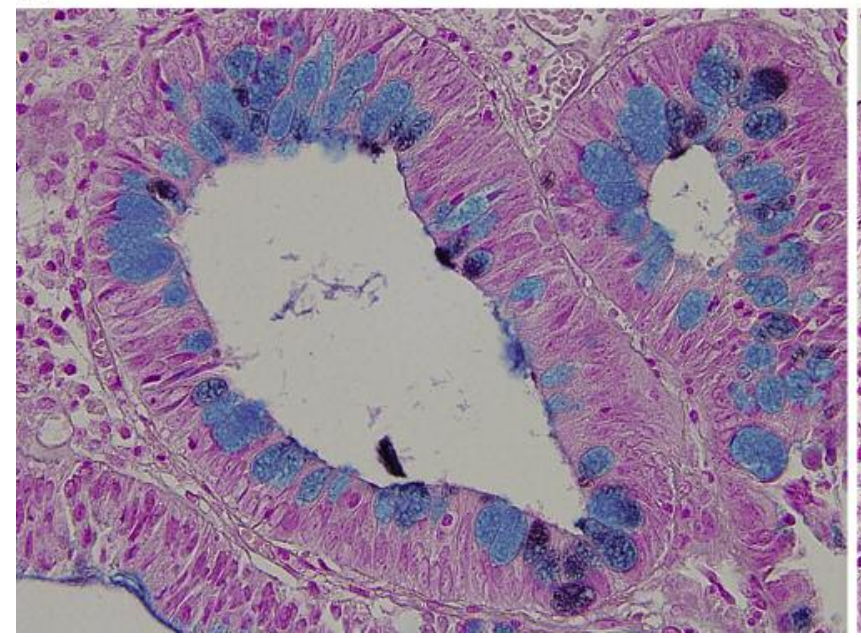

b

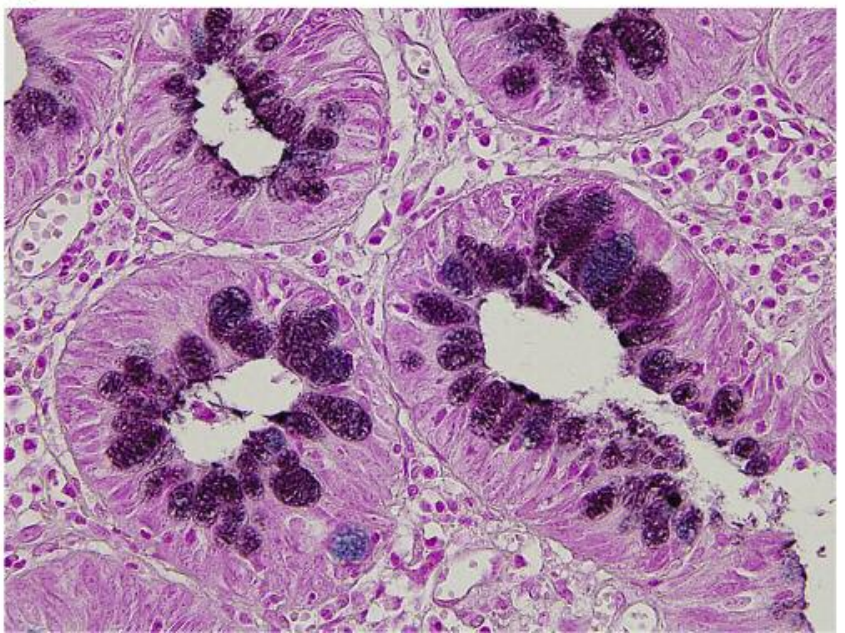

Figure 1. High iron diamine-alcian blue staining for tumor cells. (a) Example of AB>HID staining of intracytoplasmic mucin of cancer cells. Sialomucin was stained as blue. (b) Example of HID $>A B$ staining of intracytoplasmic mucin of cancer cells. Sulphomucin was stained as black.

a

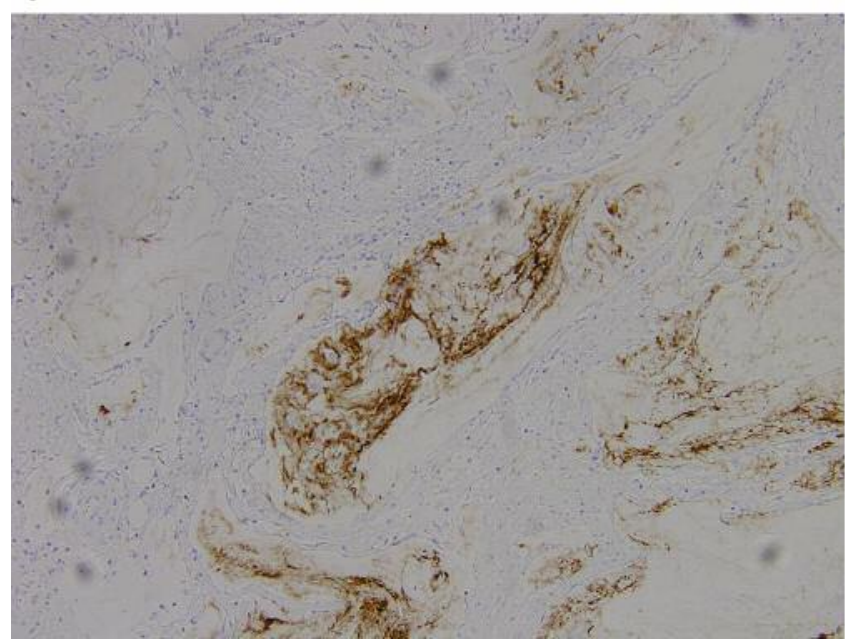

b

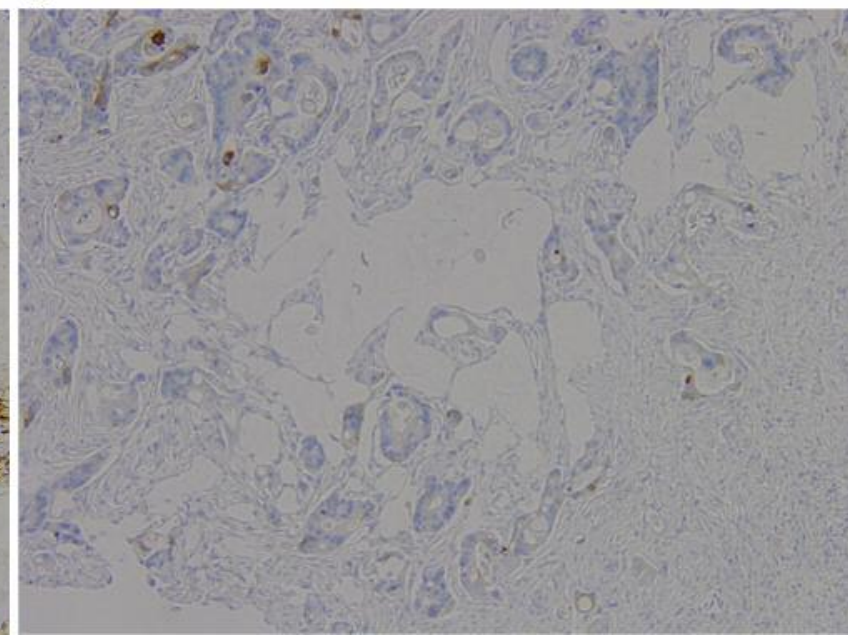

Figure 2. MUC1 Staining for Mucin Lakes. (a) Example of positive MUC1 staining. (b) Example of negative MUC1 staining.

positive for acidic mucin and are characterized by a mixture of sialomucin and sulphomucin. However, sialomucin is more abundant in MLs (18).

Ionila et al. reported that the presence of poorly differentiated cancer in mucinous carcinoma is associated with a decrease in sulphomucin and an increase in a mixture of sialomucin and sulphomucin (13). In our study, mucinous components in tumor cells were predominantly stained with $\mathrm{AB}$ in patients who received nCRT $(p=0.007)$. However, HID-AB staining of MLs did not differ between the groups.
Bergonie et al. reported that the presence of poorly differentiated cancer is associated with a high nuclear/cytoplasmic ratio, high proliferative activity, and high radiosensitivity (19). In our study, mucinous components in tumor cells were predominantly stained by $\mathrm{AB}$ rather than HID in patients who received nCRT. This is attributed to the fact that nCRT decreased the number of poorly differentiated cancer cells, thereby decreasing sulphomucin and increasing mixtures of sialomucin and sulphomucin. 
Table II. HID-AB staining and chemoradiotherapy.

\begin{tabular}{|c|c|c|c|c|c|c|}
\hline \multirow[b]{2}{*}{ Group } & \multicolumn{3}{|c|}{ Tumor cells } & \multicolumn{3}{|c|}{ MLs } \\
\hline & $\mathrm{AB}>\mathrm{HID}$ & $\mathrm{HID}>\mathrm{AB}$ & $p$-Value & $\mathrm{AB}>\mathrm{HID}$ & $\mathrm{HID}>\mathrm{AB}$ & $p$-Value \\
\hline CRT-MC & $12(60)$ & $8(40)$ & $0.0004^{*}$ & $13(65)$ & $7(35)$ & $0.6889^{*}$ \\
\hline Non-CRT & $3(11.1)$ & $24(88.8)$ & & $16(59.3)$ & $11(40.7)$ & \\
\hline Non-CRT-AC & $1(5.6)$ & $17(94.4)$ & $0.1939 \dagger$ & $9(50.0)$ & $9(50.0)$ & $0.1661^{\dagger}$ \\
\hline Non-CRT-MC & $2(22.2)$ & $7(77.8)$ & & $7(77.8)$ & $2(22.2)$ & \\
\hline
\end{tabular}

MLs: Mucin lakes; CRT: chemoradiotherapy; *CRT vs. non-CRT; † ${ }^{*}$ non-CRT-AC vs. non-CRT-MC.

Table III. MUC1 staining and chemoradiotherapy.

\begin{tabular}{|c|c|c|c|c|c|c|}
\hline \multirow[b]{2}{*}{ Group } & \multicolumn{3}{|c|}{ Tumor cells } & \multicolumn{3}{|c|}{ MLs } \\
\hline & MUC1- & MUC1+ & $p$-Value & MUC1- & MUC1+ & $p$-Value \\
\hline CRT-MC & $3(15)$ & $17(85)$ & $0.4039 *$ & $6(30)$ & $14(70)$ & $0.0254^{*}$ \\
\hline Non-CRT & $2(7.4)$ & $25(92.6)$ & & $17(63)$ & $10(37.0)$ & \\
\hline Non-CRT-AC & $0(0)$ & $18(100)$ & $0.0377^{\dagger}$ & $12(66.7)$ & $6(33.3)$ & $0.5730^{\dagger}$ \\
\hline Non-CRT-MC & $2(22.2)$ & $7(77.8)$ & & $5(55.6)$ & $4(44.4)$ & \\
\hline
\end{tabular}

MLs: Mucin lakes; CRT: chemoradiotherapy; ${ }^{*}$ CRT $v s$. non-CRT; ${ }^{\dagger}$ non-CRT-AC vs. non-CRT-MC.

Transmembrane mucins are stained by MUC1. MUC1 is expressed in colorectal cancer, and patients with MUC1 expression have been reported to have poor outcomes (14-16). MUC1 is a molecule that is induced by irradiation. Irradiation of the human colorectal cancer cell line HT29 has been reported to increase the rate of positive staining for MUC1 in cancer cells in a radiation-dose-related manner (20).

In our study, the rate of positive staining for MUC1 in MLs was $70 \%$ in patients who received CRT and $37 \%$ in those who did not, suggesting that nCRT induced MUC1 expression in MLs. The rate of positive staining for MUC1 in cancer cells was higher in the non-CRT-AC group than in the non-CRT-MC group. The difference between the groups was the quantitative difference in mucinous nodules in the maximum cut surface of tumor sections. Therefore, the reasons for the difference in the rate of positive staining for MUC1 in cancer cells remain unclear.

In our previous study, we measured the area ratio of MLs in rectal cancer tissue by magnetic resonance imaging and reported that the area ratio of MLs did not largely differ before and after nCRT and that the amount of MLs was largely unchanged after nCRT, compared to that before nCRT (21).

In the present study, we examined whether nCRT can induce qualitative changes in mucinous components in cancer tissue. However, because our study had a small sample size, it was difficult to evaluate whether nCRT induced mucinous components in cancer cells and MLs in cancer stromal tissue based only on the results of staining by
HID-AB and MUC1. Further studies are needed to confirm our results by using other diagnostic methods in larger numbers of patients.

\section{Conclusion}

In patients with rectal cancer who received nCRT, mucinous components in cancer cells were predominantly stained with $\mathrm{AB}$, and the rate of positive MUC1 staining for MLs was higher than that in patients with rectal cancer who did not receive nCRT. These findings suggested that nCRT induced quantitative changes in mucinous components. However, based only on the results of two staining methods, it is difficult to distinguish mucinous components induced by nCRT from mucinous components that were originally present.

\section{Conflicts of Interest}

H. Miyakita, S. Sadahiro, Y. Kamei, T. Ogimi, G. Saito, K. Okada, A. Tanaka, T. Suzuki, H. Kajihara and T. Akiba have no potential conflicts of interest to report. None of the Authors received any funding support for this work.

\section{References}

1 Bosset JF, Collette L, Calais G, Mineur L, Maingon P, Radosevic-Jelic L, Daban A, Bardet E and Beny A: Chemotherapy with preoperative radiotherapy in rectal cancer. N Engl J Med 355: 1114-1123, 2006. 
2 Peeters KC, Marijnen CA, Nagtegaal ID, Kanenbarg EK, Putter H, Wiggers T, Rutten H, Pahlman L, Glimeluis B, Leer JW and van de Velde: The TME trial after a median follow-up of 6 years: increased local control but no survival benefit in irradiated patients with resectable rectal carcinoma. Ann Surg 246: 693701, 2007.

3 Sauer R, Liersch T, Merkel S, Fietkau R, Hohenberger W, Hess C, Becker H, Raab HR, Villanueva MT, Witzigmann $H$, Wittekind C, Beissbarth T and Rodel C: Preoperative versus postoperative chemoradiotherapy for locally advanced rectal cancer: results of the German CAO/ARO/AIO-94 randomized phase III trial after a median follow-up of 11 years. J Clin Oncol 30: 1926-1933, 2012.

4 National Comprehensive Cancer Network: Clinical Practice Guidelines in Oncology. Rectal Cancer v.3. 2017. https:// www.nccn.org/professionals/physician_gls/pdf/rectal.pdf (accessed July 1, 2017).

5 Hugen N, van de Velde CJ, Bosch SL, Futterer JJ, Elferink MA, Marijnen CA, Rutten HJ, de Wilt JH and Nagtegaal ID: Modern treatment of rectal cancer closes the gap between common adenocarcinoma and mucinous carcinoma. Ann Surg Oncol 22: 2669-2676, 2015.

6 Yu SK, Chand M, Tait DM and Brown G: Magnetic resonance imaging defined mucinous rectal carcinoma is an independent imaging biomarker for poor prognosis and poor response to preoperative chemoradiotherapy. Eur J Cancer 50: 920-927, 2014.

7 Simha V, Kapoor R, Gupta R, Bahl A and Nada R: Mucinous adenocarcinoma of the rectum: a poor candidate for neoadjuvant chemoradiation? J Gastrointest Oncol 5: 276-279, 2014

8 Oberholzer K, Junginger T, Heintz A, Kreft A, Hansen T, Lollert A, Ebert $M$ and Duber C: Rectal Cancer: MR imaging of the mesorectal fascia and effect of chemoradiation on assessment of tumor involvement. JMRI 36: 658-663, 2012.

9 Shin US, Yu CS, Kim HJ, Kim TW, Lim SB, Yoon SN, Yoon YS, Kim CW and Kim JC: Mucinous rectal cancer: effectiveness of preoperative chemotherapy and prognosis. Ann Surg Oncol 18: 2232-2239, 2011.

10 Grillo-Ruggieri F, Mantello G, Beradi R, Cardianali M, Fenu F, Iovini G, Montisci M, Fabbietti L, Marmorale C, Guerrieri M, Saba V, Bearzi I, Mattioli R, Bonsignori M and Cascinu S: Mucinous rectal adenocarcinoma can be associated to tumor downstaging after preoperative chemoradiotherapy. Dis Colon Rectum 50: 1594-1603, 2007.

11 Sengul N, Wexner SD, Woodhouse S, Arrigain S, Xu M, Larach JA, Ahn BK, Weiss EG, Nogueras JJ and Berho M: Effects of radiotherapy on different histopathological types of rectal carcinoma. Colorectal Dis 8: 283-288, 2006.
12 Nagtegaal ID, Marijnen CA, Kranenbarg EK, Mulder-Stapel A, Hermans J, van de Velde CJ and van Krieken JH: Short-term preoperative radiotherapy interferes with the determination of pathological parameters in rectal cancer. J Pathol 197: 20-27, 2002.

13 Ionila M, Margaritescu CL, Pirici D and Mogoanta SS: Mucinous adenocarcinoma of colon - a histochemical study. Rom J Morphol Embryol 52: 783-790, 2011.

14 Khanh DT, Mekata E, Mukaisho K, Sugihara H, Shimizu T, Shiomi H, Murata S, Naka S, Yamamoto H, Endo Y and Tani T: Transmembrane mucin MUC1 overexpression and its association with $\mathrm{CD} 10+$ myeloid cells, transforming grouth factor- $\beta 1$ expression, and tumor budding grade in colorectal cancer. Cancer Sci 104: 958-964, 2013.

15 Duncan TJ, Watson NF, Al-Attar AH, Scholefield JH and Durrant LG: The role of MUC1 and MUC3 in the biology and prognosis of colorectal cancer. World J Surg Oncol 5: 31, 2007.

16 Byrd JC and Bresalier RS: Mucins and mucin binding proteins in colorectal cancer. Cancer Merast Rev 23: 77-99, 2004.

17 Baldus SE, Monig SP, Huxel S, Landsberg S, Hanisch FG, Engelmann K, Schneider PM, Thiele J, Holscher AH and Dienes HP: MUC1 and nuclear $\beta$-catenin are coexpressed at the invasion front of colorectal carcinomas and are both correlated with tumor prognosis. Clin Cancer Res 10: 2790-2796, 2004.

18 Ono K and Katsuyama T: Mucosubstance histochemistry of the normal mucosa and epithelial neoplasms of large intestine. Acta Pathol Jpn 35: 281-297, 1985.

19 Bergonie J and Tribondeau L: Interpretation of some results from Radiotherapy and attempt to determine a rational treatment technique. Radiation Res 11: 587-588, 1959.

20 Kang Y, Hirano K, Suzuki N, Enomoto A, Morita A, Irimura T and Sakai K: Increased expression after X-Irradiation of MUC1 in cultured human colon carcinoma HT-29 cells. Cancer Res 91: 324-330, 2000.

21 Miyakita H, Sadahiro S, Ogimi T, Saito G, Okada K, Tanaka A, Suzuki T, Kajiwara H, Yamamuro H and Akiba T: Mucinous components assessed by magnetic resonance imagin in primary rectal cancer tissue before and after chemoradiotherapy and tumor response. Int J Colorectal Dis, 2018. doi: 10.1007/s00384018-3047-1. [Epub ahead of print]
Received June 6, 2018

Revised June 15, 2018

Accepted June 18, 2018 